\title{
Monofocal femoral subacute neuropathy as a key diagnostic element in a case of sigmoid neoplasm
}

\author{
Ovidiu-Lucian Bajenaru ${ }^{1,2}$, Gabriel loan Prada ${ }^{1,2}$ \\ 1"Carol Davila" University of Medicine and Pharmacy, Bucharest, Romania \\ 2"Ana Aslan" National Institute of Gerontology and Geriatrics, Bucharest, Romania
}

\begin{abstract}
In this paper we present the case of a 91 years-old male patient who was admitted for motor weakness accompanied by neuropathic pain suggestive for a femoral mononeuropathy which onset and evolution was subacute - chronic. The clinical examination and laboratory investigations confirmed the diagnosis of femoral nerve mononeuropathy, but also showed others signs and symptoms suggestive for a disorder in the area in the lower digestive and urinary tracts, which finally allowed us the etiologic diagnosis of recto-sigmoid colon cancer. This case raises some problems related to the etiological differential diagnosis of a femoral nerve neuropathy which most often is not the consequence of a disco-vertebral pathology but, of other lesions located inside the pelvis as long as this nerve has its initial trajectory in the intra-abdominal region having different neighboring reports on the left and on the right side, which are also different according to the patient's sex in particular with different segments of the lower digestive tract and in women also with the salpingian-ovarian structures. Comorbidities, in particular diabetes mellitus and also the particularities related to the patient's age increase the complexity of the problem related to the etiological differential diagnosis.
\end{abstract}

Keywords: femoral nerve mononeuropathy, lower digestive tract, rectosigmoid cancer, diabetes mellitus

We present the case of a 91 years old male patient who was admitted in our department for subacute pain on the anterior aspect of the left thigh which onset was insidious some weeks before hospitalization, inferior bowel disorders (fecal evacuation with increased frequency, in small quantities and increased mucous content), frequent micturition and increased fatigability. From the personal medical history we mention a left arthroplasty for coxarthrosis three years before hospitalization, but with good recovery and without pain or motor deficiencies; he also has heart failure NYHA class I, stable coronary heart disease, hypertension with very high additional risk, diabetes mellitus type 2 , moderate iron deficiency anemia and mild neurocognitive impairment. On objective clinical examination we noticed a slight modified general condition with class 1 obesity $\left(\mathrm{BMI}=30.6 \mathrm{~kg} / \mathrm{m}^{2}\right)$, skin pallor, left hip region scar (post arthroplasty), fecal impaction, increased frequency of micturition, normal cardiovascular and thoracic clinical examination, mild muscle weakness of the left iliopsoas and quadriceps (MRC grade 3/5), abolished patellar reflex on the left side and diminished on the right side, skin hyperesthesia and dysesthesia on the anterior aspect of the left thigh and medial aspect of the left leg, very suggestive for neuropathic pain in the left femoral nerve territory without any other significant abnormality at the neurologic examination.

Among the laboratory tests we noticed a mild iron deficiency anemia (RBC $8.9 \times 10^{6} / \mu \mathrm{L}, \mathrm{Hb} 8.9$ $\mathrm{g} / \mathrm{dl}$, Htc $26.70 \%$, plasmatic iron $28 \mu \mathrm{g} / \mathrm{dL}$ ) increased ESR $60 \mathrm{~mm} / \mathrm{h}$, seric glycaemia $102 \mathrm{mg} / \mathrm{dl}$ (under treatment with gliquidone $15 \mathrm{mg}$ /day), slight increased serum creatinine $1.76 \mathrm{mg} / \mathrm{dl}$ with de- 
creased GFR $35.38 \mathrm{ml} / \mathrm{min}$ (Cockroft method), the urine culture positive for E. coli.

Our clinical diagnosis based on these data was in the initial stage: left femoral hyperalgic mononeuropathy, iron deficiency anemia, bowel elimination disturbances with fecal impaction, urinary infection with E. coli manifested by increased frequency of micturition, and biological inflammatory syndrome.

Based on this symptomatic association of diagnoses, we proceeded to an etiologic differential diagnosis including the followings: diabetic neuropathy (manifested as subacute femoral neuropathy and possible dysautonomic neuropathy), post-surgery femoral neuropathy after coxofemoral arthroplasty, subacute enterocolitis with secondary urinary tract infection with E. coli (urine culture positive for E. coli) or a neoplastic disease in the region of the lower pelvis with secondary lesions in the intra-abdominal segment in the left femoral nerve.

The hypothesis of diabetic neuropathy (1) could be excluded for the following reasons: the subacute femoral neuropathy is rare in patients with type 2 diabetes mellitus, with good glycemic control and when if it is the case, it is more acute and triggered by an abrupt decrease in glycemic levels, which was not the case in this patient; the diabetic dysautonomic neuropathy with enteral impairment is usually manifested as nocturnal diarrhea and the fecal impaction is not characteristic.

The post-surgery femoral mononeuropathy (1) after coxofemoral arthroplasty, is clinically manifest immediately after surgery (usually in such cases it has been described as being determined by surgical devices mechanical compression) and it does not appear after a delayed latency.

We excluded a subacute enterocolitis as the patient did not have signs of severe infection, fecal impaction is not a feature of an infective colitis and the patient had an enhanced biological inflammatory syndrome without clinical signs of infection.

The most probable hypothesis was a neoplastic disease in the lower pelvis which seems to be the most appropriate condition able to combine all the significant abnormal data seen in this patient, having a subacute-chronic evolution: left femoral hyperalgic mononeuropathy (by compression/infiltration on the intrapelvic segment of the femoral nerve), iron deficiency anemia (often present in neoplastic diseases), bowel elimination disturbances with fecal impaction (often associated with a neoplastic pathology of the rectosigmoid segment by progressive obstructive infiltrative mechanism), biological inflammatory syndrome (typically but not specifically associated with neoplastic pathology) and increased micturition frequency (by local compression/infiltration of the urinary bladder).

If we analyze the topographic anatomy of the femoral nerve (2-4) we have to emphasize some particularities which could be related to the particularities to this case:

- this nerve has not a direct contact with the hip joint, as it has an intra-abdominal trajectory;

- there are few published reports (5) describing a traumatic femoral neuropathy during surgery for arthroplasty but only when intraoperative lesions have been produced during the surgery and in these cases, the neuropathy manifested clinically immediately postsurgery and never after a delayed latency;

- the anatomical neighborhood reports of the femoral nerve with the organs in the lower part of the pelvis are not the same on the left and on the right side (on the left side these reports are with the descending and rectosigmoid colon and their satellite lymph nodes along the inferior mesenteric artery, and on the right side its neighborhood reports are with the caecum) (2-4);

- the sensory skin territories of this nerve (3) refers to the anterior aspect of the thigh and medial aspect of the leg (which was similar to the skin territory where our patient had typical signs of neuropathic pain).

Based on this hypothesis, we proceeded to look for direct and indirect laboratory data for a neoplasm in the area of the rectosigmoid colon. We found an increased value of the CEA (carcinoembryonic antigen) $-11.8 \mathrm{ng} / \mathrm{mL}$ (normal values $<3.0$ $\mathrm{ng} / \mathrm{mL}$ for non-smokers and $<5.0 \mathrm{ng} / \mathrm{mL}$ for smokers). The seric values if this antigen are frequently increased in cases of large bowel neoplasia (6). The CA 19-9 (carbohydrate antigen) which is also known to be increased in many patients with large bowel neoplasia (7) was normal in our patient, as also was the alkaline phosphatase and the abdomi- 
nal echography which showed non-significant findings.

Our diagnosis hypothesis has been confirmed by the abdominal CT examinations showing: asymmetric parietal increased thickness (maximum 23 $\mathrm{mm}$ ) with stenosing effect at the level of the distal part of the sigmoid colon, including the rectosigmoid junction, extending for $8 \mathrm{~cm}$ and localized at $17 \mathrm{~cm}$ to the anal verge; this lesion associated diffuse infiltration in the adjacent pericolic fat; at the same level there were some larger lymph nodes with perisigmoid topography extending in the perivascular space on the trajectory of the inferior mesenteric artery close to its aortic origin; the maximum size of these lymph nodes was $8 \times 5 \mathrm{~mm}$.

The conclusion of the CT examination was that the patient had an infiltrative stenosing sigmoid tumor with regional adenopathies.

\section{DISCUSSIONS}

The particularity of the case is the atypical onset of a rectosigmoid neoplasm in an old patient, without the classical clinical signs of a consumptive disease. Clinically, this pathology manifested atyp- ically, as a symptomatic hyperalgic femoral nerve mononeuropathy (which is quite rare) (5), accompanied by progressive fecal impaction. Among the biological markers for neoplastic gastro-enteral tract, only the CEA had increased significant levels; CEA is one of the most sensitive (6), but not very specific neoplastic markers. During the follow-up of the patient, who meanwhile had not been oncologic - specifically treated, the evolution has been very slow, with a mild general clinical deterioration accompanied by the decrease of the frequency of bowel elimination during the next 2-3 months; this is suggestive for a continuous slow progression of the infiltrative tumor in the rectosigmoid colon wall. This particular initial slow and mild clinical evolution of neoplastic diseases for a quite long delay, with an atypical clinical onset is more often seen in older patients than in younger ones (who usually have a more rapid deteriorative evolution).

As such cases are probable very rare, there are only few recent reports related to such clinical situations in the recent international literature (8-11), but we consider it as a good example for a good clinical rationale in similar cases.
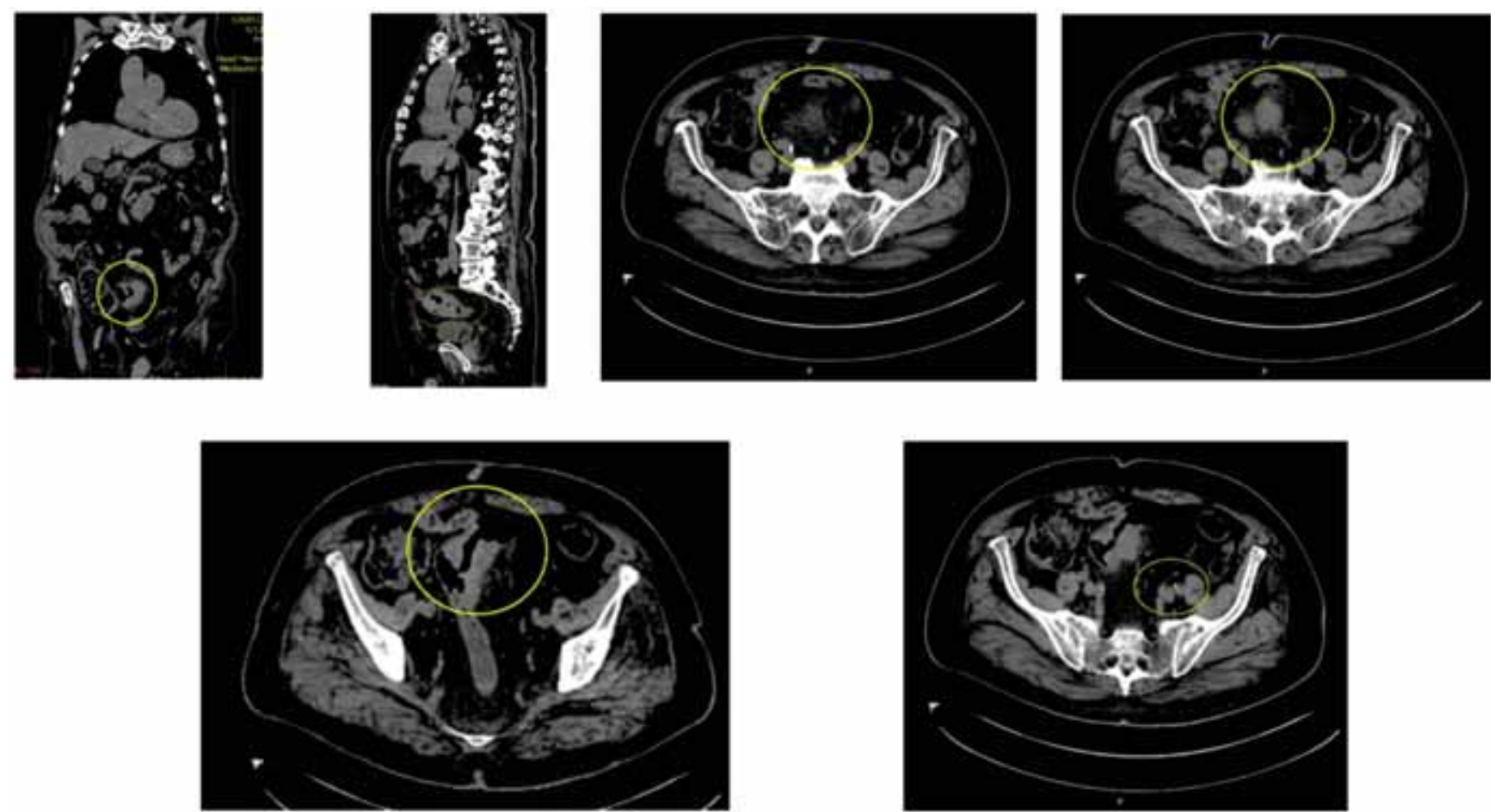

FIGURE 1. CT abdomino-pelvic examination of the patient showing the aspect of neoplastic infiltration in the wall of the rectosigmoid colon. 


\section{Acknowledgements}

Assoc. Prof. Andreea Marinescu MD, PhD - Department of Radiology, University of Medicine and
Pharmacy "Carol Davila" Bucharest, University Emergency Hospital Bucharest.

Conflict of interest: none declared Financial support: none declared

\section{REFERENCES}

1. Ropper A.H., Samuels M.A., Klein J.P., editors. Diseases of the peripheral nerves. In: Adams and Victor's Principles of Neurology. 10th ed. Mc Graw Hill Education; 2014. p. 1310-90.

2. Borley N. Posterior abdominal wall and retroperitoneum. In: Standring S, editor. Gray's Anatomy Anatomical basis of clinical practice. 39th ed. Elsevier Churchill Living Stone; 2005. p. 1113-26.

3. Newell R., Davies M. General organization and surface anatomy of the lower limb. In: Standring S, editor. Gray's Anatomy Anatomical basis of clinical practice. $39^{\text {th }}$ ed. Elsevier Churchill Living Stone; 2005. p. 1399-418.

4. Newell R., Davies M. Pelvic girdle, glutheal region and hip joint. In: Standring S, editor. Gray's Anatomy Anatomical basis of clinical practice. 39th ed. Elsevier Churchill Living Stone; 2005. p. 1419-59.

5. Geiger D., Mpinga E., Steves M.A., Sugarbaker P.H. Femoral neuropathy: unusual presentation for recurrent large-bowel cancer. Dis Colon Rectum. 1998; 41(7):910-3.

6. Livingstone A.S., Hampson L.G., Shuster J., Gold P., Hinchey E.J. Carcinoembryonic antigen in the diagnosis and management of colorectal carcinoma. Current status. Arch Surg. 1974; 109(2):259-64.
7. Zhao J-X, Liu L-R, Yang X-Y, Liu F, Zhang Z-G. Serum CA19-9 as a marker of circulating tumor cells in first reflux blood of colorectal

8. Lee K.H., Choe J.H., Lee S.E., Park J.H., Bang S.R., Kim Y.H. et al. Lumbar plexopathy caused by metastatic tumor, which was mistaken for postoperative femoral neuropathy. Korean J Pain. 2011; 24(4):226-30.

9. Yi T.I., Yoon T.H., Kim J.S., Lee G.E., Kim B.R. Femoral neuropathy and meralgia paresthetica secondary to an iliacus hematoma. Ann Rehabil Med. 2012; 36(2):273-7.

10. Genc H., Balaban O., Karagoz A., Erdem H.R. Femoral neuropathy in a patient with rheumatoid arthritis. Yonsei Med J. 2007; 48(5):891-3.

11. Desmarais A., Descarreaux M. Diagnosis and management of "an apparent mechanical" femoral mononeuropathy: a case study. J Can Chiropr Assoc. 2007; 51(4):210-6. cancer patients. Oncotarget. 2017; 8(40):67918-32. 